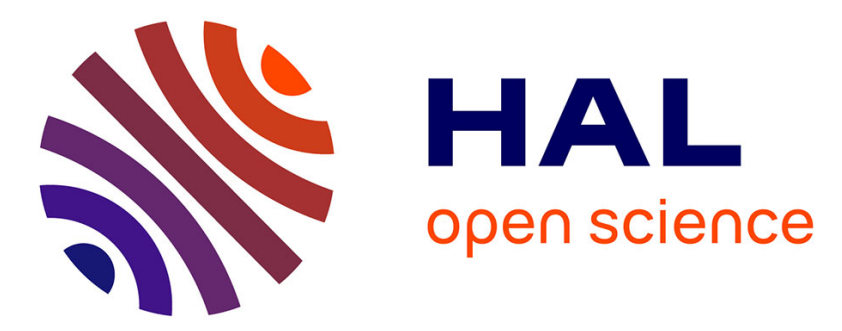

\title{
Effets galvanomagnétiques dans les semiconducteurs anisotropes inhomogènes Application à la caractérisation des films de silicium sur saphir
}

S. Cristoloveanu, J.H. Lee, A. Chovet

\section{- To cite this version:}

S. Cristoloveanu, J.H. Lee, A. Chovet. Effets galvanomagnétiques dans les semiconducteurs anisotropes inhomogènes Application à la caractérisation des films de silicium sur saphir. Revue de Physique Appliquée, 1980, 15 (3), pp.725-732. 10.1051/rphysap:01980001503072500 . jpa-00244782

HAL Id: jpa-00244782

https://hal.science/jpa-00244782

Submitted on 1 Jan 1980

HAL is a multi-disciplinary open access archive for the deposit and dissemination of scientific research documents, whether they are published or not. The documents may come from teaching and research institutions in France or abroad, or from public or private research centers.
L'archive ouverte pluridisciplinaire HAL, est destinée au dépôt et à la diffusion de documents scientifiques de niveau recherche, publiés ou non, émanant des établissements d'enseignement et de recherche français ou étrangers, des laboratoires publics ou privés. 


\title{
Effets galvanomagnétiques dans les semiconducteurs anisotropes inhomogènes Application à la caractérisation des films de silicium sur saphir
}

\author{
S. Cristoloveanu, J. H. Lee et A. Chovet \\ Laboratoire de " Physique des Composants à Semiconducteurs " $\left({ }^{*}\right)$ \\ ENSER, 23, avenue des Martyrs, 38031 Grenoble Cedex, France
}

(Reçu le 24 octobre 1979, révisé le 22 novembre 1979, accepté le 27 novembre 1979)

\begin{abstract}
Résumé. - Nous étudions du point de vue théorique les effets galvanomagnétiques dans des semiconducteurs anisotropes présentant des inhomogénéités unidirectionnelles de la masse effective, du temps de relaxation ou du dopage. En particulier, nous déduisons les relations qui gouvernent l'effet Hall et les effets de magnétorésistance longitudinale et transversale, dans une très large gamme d'inductions magnétiques. Nos résultats sont appliqués à la caractérisation des couches minces de Silicium (type n) Sur Isolant, où l'inhomogénéité est dans la direction de l'épitaxie.

La comparaison avec les expériences permet de trouver le coefficient d'anisotropie du film et de déterminer pour divers modèles plausibles d'inhomogénéité, les mobilités à l'interface $\mathrm{Si}_{-} \mathrm{SiO}_{2}$, les densités de porteurs et les mobilités moyennes.
\end{abstract}

\begin{abstract}
The galvanomagnetic effects have been theoretically analyzed for anisotropic semiconductors where the effective mass, the relaxation time and the carrier concentration, show one-directional inhomogeneities. The particular relationships defining the Hall effect, the transverse and the longitudinal magnetoresistance in a wide range of magnetic inductions are given. Our results are especially applied to the characterization of n-type Silicon On Sapphire thin films, where the non-uniformity occurs along the epitaxial direction.

The comparison with the experiments allows to determine firstly the anisotropy coefficient of the film and secondly, for different inhomogeneous models, the carrier mobility at $\mathrm{Si}_{-} \mathrm{SiO}_{2}$ interface, the carrier concentration and the mean value of the mobility.
\end{abstract}

1. Introduction. - L'utilisation de plus en plus fréquente en microélectronique de couches minces semiconductrices pose le problème de la caractérisation de matériaux inhomogènes où, en particulier, la valeur des paramètres de transport ou de recombinaison n'est pas constante selon l'épaisseur. Tel est le cas du Silicium Sur Isolant (SSI, ou SOS : Silicon On Sapphire), matériau privilégié des circuits submicroniques (VLSI). Des analyses par spectroscopie y ont montré l'existence d'un profil de défauts [1]; l'utilisation de l'effet magnétodiode a permis l'étude du gradient de durée de vie des porteurs dans la direction épitaxiale [2]; des profils de mobilité ont pu être établis par mesure d'effet Hall, ou de capacité et transconductance de MOSFET en déplétion, grâce à l'action d'une grille de contrôle $[3,4,5]$.

Dans cet article, nous présentons une étude théorique originale des effets galvanomagnétiques dans un semiconducteur présentant une inhomogénéité uni-

(*) ERA au C.N.R.S. no 659 . dimensionnelle. Nous traiterons, en particulier, l'effet Hall et les effets de magnétorésistance transversale et longitudinale en tenant compte des anisotropies de la masse effective et du temps de relaxation; nos exemples seront relatifs à du Silicium de type n. Nous montrerons comment les résultats expérimentaux obtenus sur SSI, que nous présentons ci-dessous, permettent grâce aux résultats de notre étude théorique, une analyse des anisotropies dans le SSI, et des profils de mobilité des porteurs.

\section{Résultats expérimentaux. -2.1 CondITIONS} EXPÉRIMENTALES. - Les structures Silicium Sur Isolant, dont la géométrie et les dimensions sont indiquées en figure 1, ont été réalisées au LETI-MEA (Grenoble) par croissance en phase vapeur (hétéroépitaxie par pyrolyse du silane) d'une couche de $0,65 \mu \mathrm{m}$ de silicium sur substrat de corindon $\left(\mathrm{Al}_{2} \mathrm{O}_{3}\right)$. Les dopages $\left(\mathrm{n}\right.$ et $\mathrm{n}^{+}$ ici) sont obtenus par implantation ionique ; la conductivité de la couche Si-n est d'environ $60 \Omega^{-1} \mathrm{~m}^{-1}$.

Les mesures des tensions Hall (entre $\mathrm{T}_{3}-\mathrm{T}_{4}$ et 

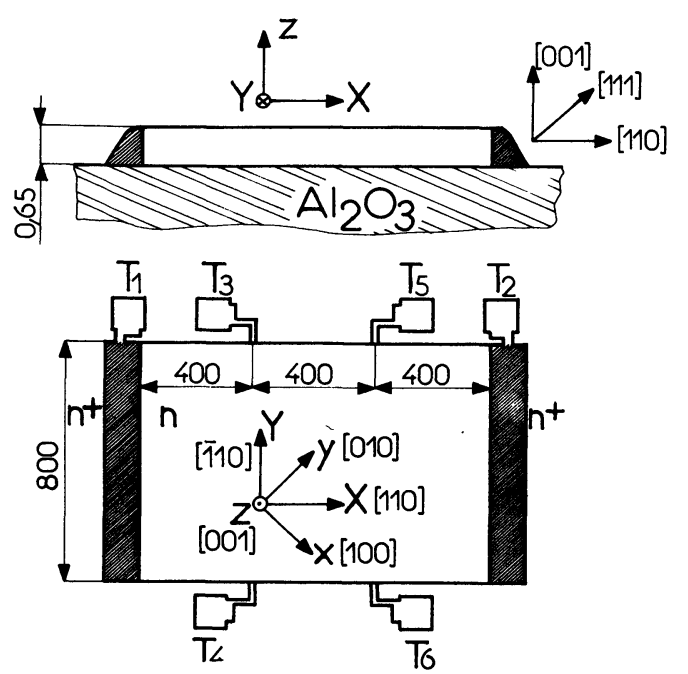

Fig. 1. - Motif de la structure à effet Hall réalisée sur SSI ; les dimensions sont données en $\mu \mathrm{m}$. Les axes $(x, y, z)$ correspondent aux directions cristallographiques principales et les coordonnées $(X, Y, z)$ aux champs extérieurs appliqués

[Silicon On Sapphire Hall device (dimensions in $\mu \mathrm{m}) .(x, y, z)$ axis correspond to crystallographic directions and $(X, Y, z)$ coordinates are related to the applied fields.]

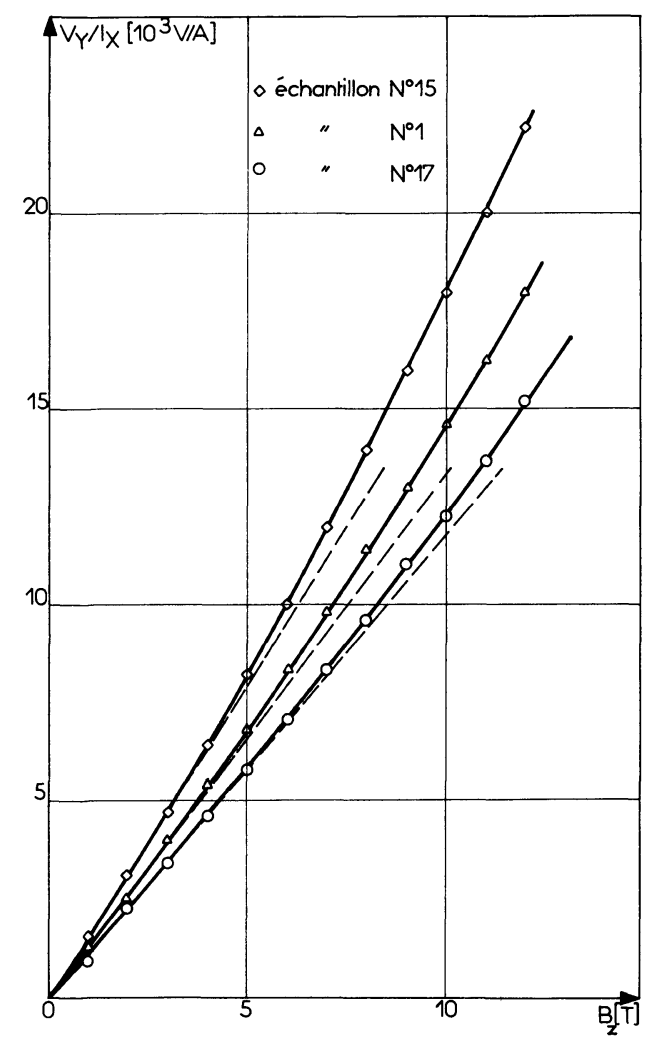

Fig. 2 - Tension Hall $V_{Y}$ en fonction de linduction magnétique $B_{z}$, relevée à courant constant $\left(I_{X}=2,5 \mu \mathrm{A}\right)$ Les lignes pointillées représentent la variation linéaire correspondant au cas des faibles inductions.

[Hall voltage $V_{Y}$ as a function of the magnetic induction $B_{z}$ (for a constant current : $I_{X}=2.5 \mu \mathrm{A}$ ). The dashed lines give the linear variation following the slope for low inductions.]
$T_{5}-T_{6}$ ) et des tensions longitudinales (entre $T_{1}-T_{2}$, $T_{3}-T_{5}$ et $T_{4}-T_{6}$ ) ont été effectuées pour les divers sens des champs électrique et magnétique, la structure étant parcourue par un courant constant et faible $\left(I_{X}<3 \mu \mathrm{A}\right)$. Les combinaisons convenables des diverses valeurs obtenues permettent d'éliminer les risques d'erreur systématique (due à des dissymétries des contacts, aux jonctions $\left.\mathrm{n}^{+}-\mathrm{n}, \ldots\right)$. Les caractéristiques ont été relevées à température ambiante $(290 \mathrm{~K})$, les inductions magnétiques $(B \leqslant 15 \mathrm{~T})$ étant obtenues dans des bobines de Bitter.

2.2 EfFet Hall. - Les caractéristiques des tensions Hall en fonction de l'induction magnétique $B_{z}$ relevées à courant constant $\left(I_{X}=2,5 \mu \mathrm{A}\right)$ pour divers échantillons sont présentées dans la figure 2 . Signalons qu'en raison de la géométrie de nos échantillons (faible rapport longueur $l_{X}$ sur épaisseur $l_{Y}$ ), les tensions Hall mesurées sont réduites par rapport aux tensions Hall réelles que l'on obtiendrait sur des structures longues (ceci étant dû aux effets de courtcircuit des contacts d'extrémité). Dans notre cas (pour $l_{X} / l_{Y}=3 / 2$ et compte tenu de la position géométrique des contacts Hall), les tensions Hall relevées doivent être corrigées par un facteur voisin de $4 / 3$ aux inductions faibles $\left(B_{z}<5\right.$ T) [6]; aux inductions plus élevées, l'effet de la géométrie diminue $[6,7]$ ce qui explique la surlinéarité de nos courbes $V_{Y}\left(B_{z}\right)$.

2.3 MAGNÉTORÉSISTANCES. - Les mesures de magnétorésistance ont été effectuées pour trois orientations particulières du champ magnétique :

2.3.1 Magnétorésistance transversale géométrique : $\mathbf{B}\left(0,0, B_{z}\right)$. - L'effet de géométrie observé sur l'effet Hall pour la même configuration des champs se manifeste aussi sur la magnétorésistance : la figure 3 montre que l'augmentation de résistance par application du champ magnétique est nettement moins importante dans la région centrale de l'échantillon (entre $T_{3}$ et $T_{5}$, ou $T_{4}$ et $T_{6}$ ) que sur l'ensemble de la structure (entre $T_{1}$ et $T_{2}$ ); ceci souligne que l'effet de magnétorésistance est accentué au voisinage des contacts d'extrémités, puisque ceux-ci court-circuitent le champ Hall.

2.3.2 Magnétorésistance transversale réelle : $\mathbf{B}\left(0, B_{Y}, 0\right)$. - Pour cette orientation de l'induction, la force de Lorentz se manifeste selon l'épaisseur $l_{z}$, très inférieure à la longueur $l_{X}$ : l'effet de court-circuit des contacts d'extrémités est négligeable, et la magnétorésistance prend sa vraie valeur (Fig. 4); elle dépend principalement de la dispersion statistique des vitesses des porteurs et reste relativement faible.

2.3.3 Magnétorésistance longitudinale : $\mathbf{B}\left(B_{X}, 0\right.$, $0)$. - Le courant électrique total et l'induction magnétique étant parallèles, la magnétorésistance est due à l'anisotropie de conduction électrique du matériau (le courant dans les divers ellipsoïdes de conduction n'est pas parallèle au champ électrique 


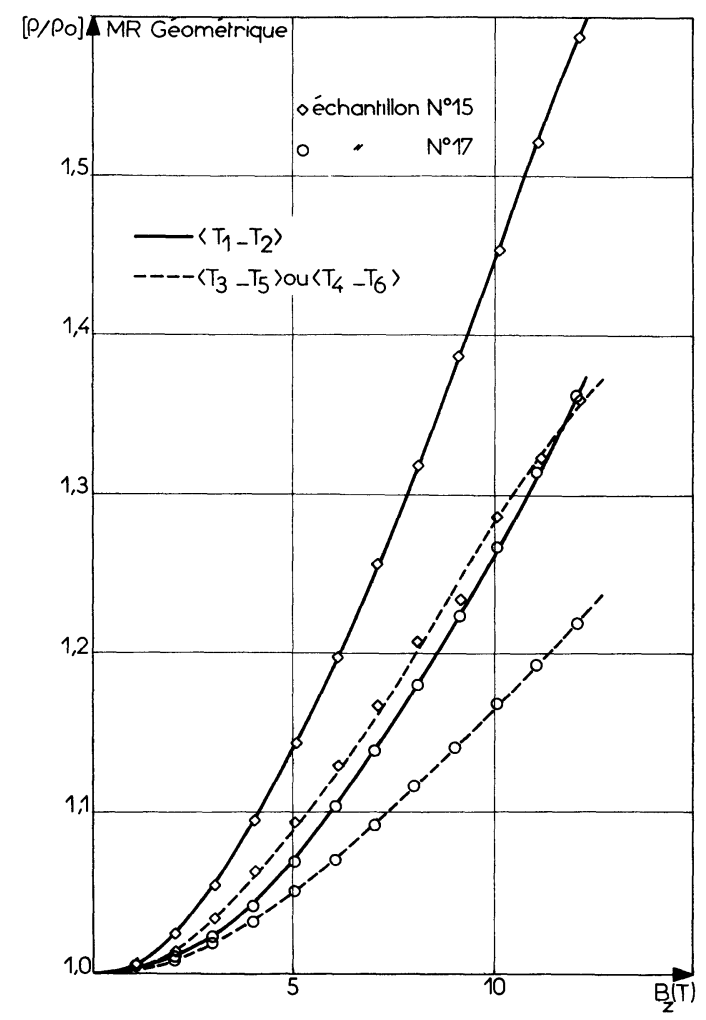

Fig. 3. - Coefficient de magnétorésistance transversale géométrique $\rho / \rho_{0}$ en fonction de l'induction magnétique $B_{z}$. Les courbes continues, relevées entre les extrémités $T_{1}$ et $T_{2}$ montrent le comportement de l'ensemble de l'échantillon; les courbes pointillées correspondent à la magnétorésistance des zones centrales de l'échantillon $\left(T_{3}-T_{5}\right.$ ou $\left.T_{4}-T_{6}\right)$, plus faiblement affectées par l'effet de court-circuit des contacts d'extrémité

[Transverse magnetoresistance coefficient $\rho / \rho_{0}$ versus magnetic induction $B_{z}$ for the field geometry $\left(E_{X} \perp B_{z}\right)$ leading to the shorting effect. The full lines (corresponding to the end contacts $T_{1}-T_{2}$ ) show the magnetoresistance of the whole sample; the dashed lines give the partial magnetoresistance of the central region $\left(\mathrm{T}_{3}-\mathrm{T}_{5}\right.$ or $T_{4}-T_{6}$ ) where the shorting effect due to the end contacts is attenuated.]

appliqué). Les résultats expérimentaux de la figure 4 dénotent donc une forte anisotropie du SSI.

3. Analyse théorique (Si-n). - Dans cette partie, avant de détailler les situations particulières engendrées par l'inhomogénéité du SSI, nous commencerons par donner l'expression du tenseur de conductivité électrique sous une induction magnétique arbitraire (non quantique) dans le silicium parfait de type $\mathrm{n}$.

3.1 MatéRIAU HOMOGÈNE. - La méthode décrite par Shibuya $[8,9$, p. 236] permet de résoudre l'équation isotherme de Boltzmann pour des inductions magnétiques d'intensité arbitraire et dans le cas d'une anisotropie de la masse effective $m$. Sous les hypothèses usuelles (approximation du temps de relaxation, surfaces isoénergétiques ellipsoïdales, faible champ électrique, courant de diffusion négligeable, pas de gradient de température), on peut alors obtenir le tenseur de conductivité. Herring et Vogt [10] ont

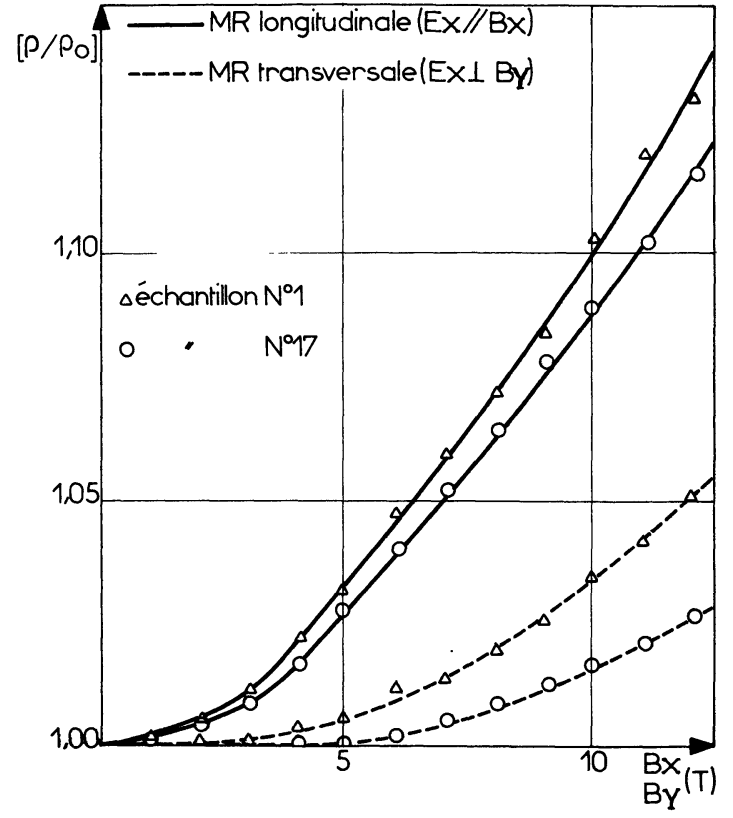

Fig. 4. - Coefficients de magnétorésistance longitudinale ou transversale réelle en fonction de l'induction magnétique $B_{X}$ ou $B_{Y}$.

[Longitudinal or transverse magnetoresistance coefficient versus magnetic induction $B_{X}$ or $B_{Y}$.]

montré par ailleurs que l'anisotropie du temps de relaxation $\tau$ se manifeste comme une pondération de l'anisotropie de masse effective; seuls interviennent dans le calcul les rapports $\tau / m$; ainsi les composantes du tenseur de conductivité $\stackrel{\boldsymbol{\sigma}}{ }^{(i)}$ pour chaque ellipsoïde (i) de la bande de conduction $[8,9$, p. 236] peuvent être aisément déterminées dans le cas, plus complet, d'une anisotropie de $m$ et de $\tau$ :

$$
\begin{aligned}
\sigma_{\alpha \beta}^{(\mathrm{i})}=\frac{n e^{2}}{6}\left(\overline{\frac{\tau_{\alpha} / m_{\alpha}}{D}}\right) & \delta_{\alpha \beta}-\frac{n e^{3}}{6} B_{\gamma} \overline{\left(\frac{\tau_{\alpha} \tau_{\beta} / m_{\alpha} m_{\beta}}{D}\right)} \delta_{\alpha \beta \gamma}+ \\
+ & \frac{n e^{4}}{6} B_{\alpha} B_{\beta}\left(\frac{\tau_{x} \tau_{y} \tau_{z} / m_{x} m_{y} m_{z}}{D}\right)
\end{aligned}
$$

où :

est la charge électrique élémentaire,

$n \quad$ la densité d'électrons libres,

$$
D=1+e^{2}\left(B_{x}^{2} \frac{\tau_{y} \tau_{z}}{m_{y} m_{z}}+B_{y}^{2} \frac{\tau_{z} \tau_{x}}{m_{z} m_{x}}+B_{z}^{2} \frac{\tau_{x} \tau_{y}}{m_{x} m_{y}}\right)
$$

$\delta_{\alpha \beta} \quad$ est le symbole de Kronecker,

$\delta_{\alpha \beta \gamma}$ étant égal à +1 pour des permutations directes, -1 pour des permutations inverses de $\alpha, \beta, \gamma$ parmi $(x, y, z)$ et 0 pour les autres cas ;

et la moyenne sur l'ensemble des énergies $\varepsilon$ d'une fonction $A(\varepsilon)$ étant définie par :

$$
(\bar{A})=\frac{\int_{0}^{\infty} \varepsilon^{3 / 2} A(\varepsilon) \exp (-\varepsilon / k T) \mathrm{d} \varepsilon}{\int_{0}^{\infty} \varepsilon^{3 / 2} \exp (-\varepsilon / k T) \mathrm{d} \varepsilon}
$$


Pour chaque ellipsoïde, on explicite ensuite selon les directions longitudinales et transversales les temps de relaxation $\left(\tau_{1}, \tau_{\mathrm{t}}=\tau\right)$ et les masses effectives $\left(m_{1}, m_{\mathrm{t}}=m\right)$; le coefficient global d'anisotropie, généralement fonction de l'énergie $\varepsilon$ est :

$$
K=\frac{m_{1}}{m} \cdot \frac{\tau}{\tau_{1}} .
$$

En sommant les contributions des 6 ellipsoïdes de la bande de conduction du silicium, on obtient alors, à partir de l'équation (1), le tenseur de conductivité pour l'ensemble des électrons :

$$
\begin{aligned}
\sigma_{\alpha \beta}=\frac{n e^{2}}{3 m} \delta_{\alpha \beta} & \overline{\left.\tau \sum_{u=x, y, z}\left(1 / D_{u}\right)+\tau\left(\frac{1}{K}-1\right) \sum_{u} \frac{\delta_{\alpha u}}{D_{u}}\right]} \\
& -\frac{n e^{3}}{3 m^{2}} B_{\gamma} \delta_{\alpha \beta \gamma} \overline{\left.\left[\tau^{2} \frac{1}{K D_{\alpha}}+\frac{1}{K D_{\beta}}+\frac{1}{D_{\gamma}}\right)\right]} \\
& +\frac{n e^{4}}{3 m^{3}} B_{\alpha} B_{\beta} \overline{\left(\frac{\tau^{3}}{K} \sum_{u} \frac{1}{D_{u}}\right)}
\end{aligned}
$$

avec :

$$
D_{x}=1+\left(\frac{e \tau}{m}\right)^{2}\left(B_{x}^{2}+\frac{B_{y}^{2}}{K}+\frac{B_{z}^{2}}{K}\right),
$$

$D_{y}, D_{z}$ s'obtenant par permutation circulaire.

A l'aide du tenseur $\overleftrightarrow{\boldsymbol{\sigma}}$ on peut expliciter tout effet galvanomagnétique, quelle que soit l'orientation de l'échantillon par rapport aux champs extérieurs. Les exemples et applications donnés ci-dessous correspondent en outre au cas plus complexe d'un matériau inhomogène.

3.2 Matériau INHOMOgÈne. - 3.2.1 Considérations générales. - L'inhomogénéité du semiconducteur, supposée unidirectionnelle selon l'axe $\mathbf{O} z$, englobe des variations de la masse effective, du temps de relaxation ou de la densité des porteurs. Nous définissons la moyenne $\langle A\rangle$ d'une grandeur $A(z)$ par :

$$
\langle A\rangle=\langle A(z)\rangle=\frac{1}{l_{z}} \int_{0}^{l_{z}} A(z) \mathrm{d} z .
$$

Loin des contacts d'extrémité, en supposant négligeable tout déséquilibre de porteurs dans un plan $z=$ Cte, le champ électrique $\mathbf{E}$ et la densité de courant $\mathbf{i}$ ne sont fonction que de $z$.

L'équation de Maxwell $\boldsymbol{\nabla} \wedge \mathbf{E}=\mathbf{0}$ et l'équation de conservation du courant $(\nabla \cdot \mathbf{i}=0)$ impliquent que, les composantes $E_{x}, E_{y}, i_{z}$ sont indépendantes de $z$.

Par suite de l'inhomogénéité, le tenseur $\overleftrightarrow{\boldsymbol{\sigma}}$ est ici fonction de $z$.

Nous allons maintenant expliciter le calcul des effets galvanomagnétiques correspondant à nos résultats expérimentaux.
3.2.2 Conductivité $(B=0)$. - Dans ce cas, $\overleftrightarrow{\sigma}$ se réduit à un scalaire $a_{0}$ :

$$
a_{0}(z)=\frac{n e^{2}}{3 m}\left(\frac{2 K+1}{K} \tau\right) .
$$

Alors : $\langle\mathbf{i}\rangle=\left\langle a_{0}(z) \mathbf{E}(z)\right\rangle$ et, si le courant total est situé dans un plan perpendiculaire à $\mathbf{O} z$, la conductivité apparente est :

$$
\sigma_{0}=\left\langle a_{0}(z)\right\rangle .
$$

Si $n$ est constant selon $z$, la mobilité moyenne mesurée est donc définie par

$$
\mu_{0}=\frac{e}{3}\left\langle\frac{1}{m} \overline{\left.\left(\frac{2 K+1}{K} \tau\right)\right\rangle .}\right.
$$

Remarquons que, selon la direction $\mathbf{O} z$, le courant $i_{z}$ étant constant, on obtiendrait : $E_{z}(z)=i_{z} / a_{0}(z)$, et donc

$$
\sigma_{0}=i_{z} /\left\langle E_{z}\right\rangle=\left(\left\langle\frac{1}{a_{0}}\right\rangle\right)^{-1} .
$$

\subsubsection{Magnétorésistance longitudinale}

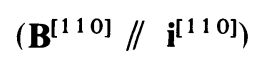

L'équation (2) conduit dans ce cas à :

$$
\mathbf{i}(z)=\left(\begin{array}{rrr}
a_{1} & a_{2} & a_{3} \\
a_{2} & a_{1} & -a_{3} \\
-a_{3} & a_{3} & a_{4}
\end{array}\right) \mathbf{E}
$$

les coefficients $a_{i}$, fonctions de $z$ pour notre matériau inhomogène, étant définis par :

$$
\begin{aligned}
& a_{1}=\frac{n e^{2}}{3 m}\left[\tau\left(\frac{K+1}{K D_{1}}+\frac{1}{D_{2}}\right)\right]+a_{2} \\
& a_{2}=\frac{n e^{4}}{6 m^{3}} B^{2}\left[\frac{\tau^{3}}{\left.K\left(\frac{2}{D_{1}}+\frac{1}{D_{2}}\right)\right]}\right. \\
& a_{3}=\frac{n e^{3}}{6 m^{2}} \sqrt{2} B\left[\frac{\tau^{2}}{K}\left(\frac{K+1}{D_{1}}+\frac{1}{D_{2}}\right)\right] \\
& a_{4}=\frac{n e^{2}}{3 m} \overline{\left[\left(\frac{2}{D_{1}}+\frac{1}{K D_{2}}\right)\right]}
\end{aligned}
$$

avec

$$
\begin{aligned}
& D_{1}=1+\frac{e^{2} \tau^{2}}{2 K m^{2}}(K+1) B^{2} \\
& D_{2}=1+\frac{e^{2} \tau^{2}}{K m^{2}} B^{2}
\end{aligned}
$$

Puisque $\left\langle i_{Y}\right\rangle=0$ et $i_{z}(z) \equiv 0$, on déduit de (4), les coordonnées $(\mathrm{X}, \mathrm{Y}, \mathrm{z})$ étant relatives à l'échantillon :

$$
\begin{gathered}
E_{x}=E_{y}=\frac{\sqrt{2}}{2} E_{X} \quad \text { et } \quad E_{z}=0 ; \\
i_{X}(z)=\frac{\sqrt{2}}{2}\left(i_{x}+i_{y}\right)=\left(a_{1}+a_{2}\right) E_{X} .
\end{gathered}
$$


En sommant les courants longitudinaux élémentaires $i_{X}$, on trouve la conductivité $\left(\sigma_{\mathrm{B}}\right)_{\mathbf{i}}^{\mathbf{B}}$ et la magnétorésistance $\left(\rho_{\mathrm{B}} / \rho_{0}\right)_{\mathbf{i}}^{\mathbf{B}}$

$$
\begin{aligned}
\left(\sigma_{\mathbf{B}}\right)_{110}^{110} & =\left\langle a_{1}+a_{2}\right\rangle \\
\left(\rho_{\mathbf{B}} / \rho_{0}\right)_{110}^{110} & =\frac{\left\langle a_{0}\right\rangle}{\left\langle a_{1}+a_{2}\right\rangle}
\end{aligned}
$$

A très haut champ magnétique, l'expression (5) se simplifie :

$$
\left(\rho_{\mathrm{B}} / \rho_{0}\right)_{110}^{110} \frac{\left\langle\frac{n}{m} \overline{\left(\frac{2 K+1}{K} \tau\right)}\right\rangle}{\left\langle\frac{n}{m} \overline{\left.\left(\frac{K+5}{K+1} \tau\right)\right\rangle}\right.} ;
$$

si le coefficient d'anisotropie est indépendant de l'énergie $\varepsilon$ et constant selon $z$, on retrouve le résultat classique (voir par exemple [9, p. 242]) :

$$
\left(\rho_{\mathbf{B}} / \rho_{0}\right)_{110}^{110} \underset{B \rightarrow \infty}{\longrightarrow} \frac{(2 K+1)(K+1)}{K(K+5)} ;
$$

qui permet dans ce cas de déterminer le coefficient d'anisotropie quelles que soient les inhomogénéités du dopage, de la masse effective ou du processus de collisions.

\subsubsection{Magnétorésistance transversale}

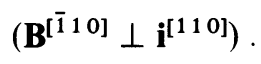

Avec les mêmes notations que précédemment, on a :

$$
\mathbf{i}=\left(\begin{array}{rrr}
a_{1} & -a_{2} & a_{3} \\
-a_{2} & a_{1} & a_{3} \\
-a_{3} & -a_{3} & a_{4}
\end{array}\right) \mathbf{E}
$$

avec

$$
i_{z}(z) \equiv 0 \quad \text { et } \quad i_{Y}(z) \equiv 0 .
$$

On en déduit $E_{x}=E_{y} ;$ la conductivité vaut :

$$
\left(\sigma_{\mathrm{B}}\right)_{110}^{\overline{1} 10}=\left\langle a_{1}-a_{2}+\frac{2 a_{3}^{2}}{a_{4}}\right\rangle ;
$$

et le champ Hall $E_{z}$ :

$$
E_{z}(z)=\sqrt{2} \frac{a_{3}}{a_{4}} \frac{\left\langle i_{X}\right\rangle}{\sigma_{\mathrm{B}}} .
$$

A haut champ magnétique, cette magnétorésistance transversale se sature :

$$
\left(\rho_{\mathbf{B}} / \rho_{0}\right)_{110}^{\overline{1} 110} \underset{B \rightarrow \infty}{\longrightarrow} \frac{\left\langle\frac{n}{m} \overline{\left.\left(\frac{2 K+1}{K} \tau\right)\right\rangle}\right.}{\left\langle\frac{9 n}{m} \frac{1}{\left[\frac{5 K+1}{(K+1) \tau}\right]}\right\rangle}
$$

si $K$ est constant et si les collisions des porteurs avec les phonons prédominent $(\bar{\tau} \cdot \overline{(1 / \tau)}=32 / 9 \pi)$, on obtient, quelles que soient les inhomogénéités de $n, \tau$ et $m$ :

$$
\left(\rho_{\mathbf{B}} / \rho_{0}\right)_{110}^{\overline{1} 10} \underset{B \rightarrow \infty}{\longrightarrow} \frac{32}{9 \pi} \frac{(2 K+1)(5 K+1)}{9 K(K+1)} ;
$$

la valeur de saturation reste en général relativement faible puisque pour $K>1$ l'augmentation relative de résistance est inférieure à $20 \%$.

3.2.5 Magnétorésistance transversale dans la configuration des effets de géométrie $\left(\mathbf{B}^{[001]} \perp \mathbf{i}^{[110]}\right)$. On obtient alors :

$$
\mathbf{i}=\left(\begin{array}{ccc}
a_{5} & a_{6} & 0 \\
-a_{6} & a_{5} & 0 \\
0 & 0 & a_{7}
\end{array}\right) \mathbf{E}
$$

avec :

$$
\begin{aligned}
& a_{5}= \frac{n e^{2}}{3 m}\left[\tau\left(\frac{K+1}{K D_{2}}+\frac{1}{D_{3}}\right)\right] \\
& a_{6}=-\frac{n e^{3}}{3 m^{2}} B\left[\tau^{2}\left(\frac{2}{K D_{2}}+\frac{1}{D_{3}}\right)\right] \\
& a_{7}=\frac{n e^{2}}{3 m}\left[\tau\left(\frac{2}{D_{2}}+\frac{1}{K D_{3}}\right)\right]+ \\
&+\frac{n e^{4}}{3 m^{3}} B^{2}\left[\frac{\tau^{3}}{K}\left(\frac{2}{D_{2}}+\frac{1}{D_{3}}\right)\right]
\end{aligned}
$$

et

$$
D_{3}=1+\frac{e^{2} \tau^{2}}{m^{2}} B^{2}
$$

D'où, après passage aux coordonnées $(X, Y, z)$ relatives à l'échantillon (cf. Fig. 1) :

$$
\left\langle i_{X, Y}\right\rangle=\left\langle a_{5}\right\rangle E_{X, Y} \pm\left\langle a_{6}\right\rangle E_{Y, X} .
$$

Le courant transversal total $\left\langle i_{Y}\right\rangle$, dans la structure, étant nul, il vient :

$$
E_{Y}=\frac{\left\langle a_{5}\right\rangle}{\left\langle a_{6}\right\rangle} E_{X}
$$

et la conductivité s'écrit :

$$
\left(\sigma_{\mathrm{B}}\right)_{110}^{001}=\left\langle a_{5}\right\rangle+\frac{\left\langle a_{6}\right\rangle^{2}}{\left\langle a_{5}\right\rangle} .
$$

A haut champ magnétique

$\left(\rho_{\mathbf{B}} / \rho_{0}\right)_{110}^{001} \underset{\mathbf{B} \rightarrow \infty}{\longrightarrow}$

$$
\frac{\left\langle\frac{n}{m} \overline{\left.\left(\frac{2 K+1}{K} \tau\right)\right\rangle\left\langle n m \overline{\left(\frac{K+2}{\tau}\right)}\right\rangle} ;\right.}{9\langle n\rangle^{2}} \text {. }
$$

si $K(\varepsilon, z), \tau(z)$ et $m(z)$ sont des constantes, la magnétorésistance se sature à une valeur indépendante du dopage $n(z)$, soit, pour des collisions avec les phonons :

$$
\left(\rho_{\mathrm{B}} / \rho_{0}\right)_{110}^{001} \underset{B \rightarrow \infty}{\longrightarrow} \frac{32}{9 \pi} \frac{(2 K+1)(K+2)}{9 K} .
$$


3.2.6 Effet Hall $\left(\mathbf{B}^{[001]} \perp \mathbf{i}^{[110]}\right)$. - La relation (7) permet de définir la mobilité de Hall :

$$
\left(\mu_{\mathrm{H}}\right)_{110}^{001}=-\frac{1}{B} \frac{\left\langle a_{6}\right\rangle}{\left\langle a_{5}\right\rangle},
$$

et la constante de Hall $\left(R_{\mathrm{H}}\right)_{i}^{\mathbf{B}}$ sera donnée par :

$$
\left(R_{\mathrm{H}}\right)_{\mathbf{i}}^{\mathbf{B}}=-\frac{\mathbf{E}(\mathbf{i} \wedge \mathbf{B})}{i^{2} B^{2}}=-\frac{\mu_{\mathrm{H}}}{\sigma_{\mathbf{B}}}
$$

Pour $B \rightarrow 0$ :

$$
\left(\mu_{\mathrm{H}}\right)_{110}^{001}=\frac{e\left\langle\frac{n}{m^{2}}\left(\tau^{2} \frac{2+K}{K}\right)\right\rangle}{\left\langle\frac{n}{m}\left(\tau \frac{2 K+1}{K}\right)\right\rangle}
$$

Pour $B \rightarrow \infty$ :

$$
\left(\mu_{\mathrm{H}}\right)_{110}^{001}=\frac{3 e\langle n\rangle}{\left\langle n m\left(\frac{K+2}{\tau}\right)\right\rangle}
$$

Il est intéressant de constater que, dans ce cas, la constante de Hall ne dépend que de liinhomogénéité du dopage; on obtient en effet :

$$
\left(R_{\mathrm{H}}\right)_{110}^{001}=-\frac{1}{\langle n\rangle e} .
$$

Remarque générale. - Dans la formulation de tout phénomène se produisant dans un plan transversal à la direction $\mathbf{O} z$ (donc où aucune force n'agit sur les porteurs selon $\mathbf{O} z$, et où aucun champ $E_{z}(z)$ n'intervient), on peut déduire les résultats relatifs à un matériau inhomogène suivant $\mathbf{O} z$ à partir de ceux relatifs à un matériau homogène en remplaçant simplement les coefficients $a_{i}$ par leurs valeurs moyennes $\left\langle a_{i}(z)\right\rangle$. Cette substitution est généralement fausse pour des phénomènes de transport faisant intervenir la direction $\mathbf{O} z$ (cf. § 3.2.4).

4. Inhomogénéités du SSI. - Nous allons appliquer les résultats théoriques précédents à l'étude des propriétés des couches de Silicium Sur Isolant dont le comportement expérimental a été présenté au paragraphe 2 .

4.1 ApProximations. - L'inhomogénéité du SSI semble due principalement aux contraintes existant à l'interface $\mathrm{Si}-\mathrm{Al}_{2} \mathrm{O}_{3}$ (provenant du désaccord entre les réseaux cristallins du silicium et du saphir) ainsi qu'à la contamination en atomes d'aluminium pendant la formation du film semiconducteur; une très forte densité de défauts a été mise en évidence sur les 500 à $700 \AA$ proches de l'interface $\mathrm{Si}_{-} \mathrm{Al}_{2} \mathrm{O}_{3}$ [2] dans des couches obtenues, comme les nôtres, par croissance en phase vapeur. Ceci nous a incité à envisager aussi pour les paramètres de transport des inhomogénéités présentant des variations al,ruptes au voisinage de l'interface avec le saphir. D'autres auteurs $[3,4,5,11]$ ont proposé ou mesuré, pour des couches de SSI obtenues par d'autres techniques, des décroissances exponentielles ou linéaires de la mobilité avec la distance à l'interface $\mathrm{Si}_{-} \mathrm{SiO}_{2}$.

Nous allons envisager et comparer aux résultats expérimentaux des effets galvanomagnétiques du paragraphe 2 , les conséquences et propriétés de deux modèles (ahrupt ou linéaire); pour limiter le nombre de paramètres à déterminer, nous supposons que :

- la mobilité des porteurs dans les $500 \AA$ près de l'interface $\mathrm{Si}-\mathrm{Al}_{2} \mathrm{O}_{3}$ est négligeable par rapport à la mobilité dans le volume;

- le dopage présente toujours un profil ahrupt, ce qui conduit aux modèles simplifiés de la figure 5 .

Nous supposons de plus, en première approximation, que le coefficient d'anisotropie $K$ ne varie pas dans l'épaisseur du film (direction $\mathbf{O} z$ ) et qu'il est indépendant de l'énergie $\varepsilon$ des porteurs (ce dernier point est rigoureusement exact si les temps de relaxation longitudinal et transversal dépendent de $\varepsilon$ de façon similaire).

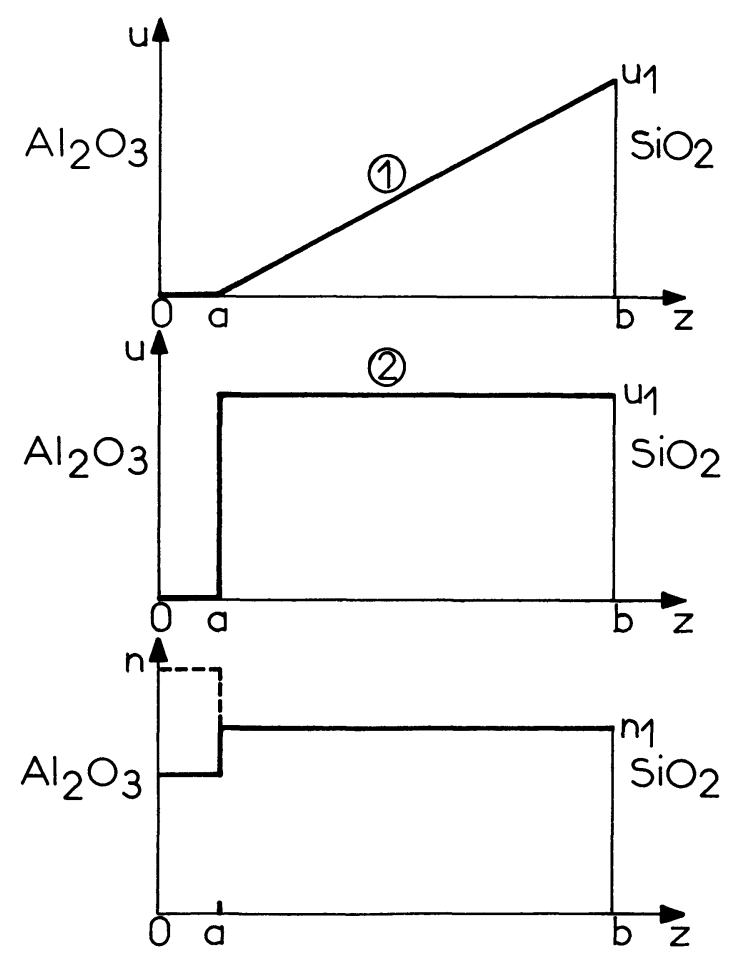

Fig. 5. - Schémas de deux modèles physiques simplifiés d'inhomogénéités dans le SSI : $u=e \bar{\tau} / m \propto \mu ; a=0,05 \mu \mathrm{m}$ : $h=0,65 \mu \mathrm{m}$; (1) : modèle linéaire ; (2) : modèle abrupt ; Dans la région active (à mobilité non nulle), le dopage est considéré comme quasi uniforme.

[Simplified models of the inhomogeneity in thin SOS films : $u=\bar{e} \bar{\tau} / m \propto \mu ; a=0.05 \mu \mathrm{m} ; b=0.65 \mu \mathrm{m}$; (1) Linear model; (2) Abrupt model; In the active region (with non-zero mobility), the doping is considered as nearly homogeneous.] 
4.2 RéSultats. - Les paramètres physiques à déterminer $\left(n_{1}, \mu_{1}\right.$ : densité et mobilité des porteurs à linterface $\mathrm{Si}_{-} \mathrm{SiO}_{2}$, et le coefficient d'anisotropie $K$ ) seront déduits des mesures de conductivité $\sigma_{0}$ (éq. (3)), d'effet Hall pour de faibles inductions (éq. (10)) et de magnétorésistance longitudinale dans une large gamme de champs magnétiques (les mesures de magnétorésistance transversale présentant dans notre cas une trop faible résolution ne sont pas utilement exploitables).

En considérant une mobilité élémentaire

$$
u(z)=e(\bar{\tau}) / m
$$

dans le plan de cote $z$, l'équation (3) permet d'écrire :

$$
\sigma_{0}=e \frac{2 K+1}{3 K}\langle n u\rangle
$$

La mobilité de Hall à bas champ magnétique (éq. (10)) devient :

$$
\left(\mu_{\mathrm{H}}\right)_{110}^{001} \underset{B \rightarrow 0}{\longrightarrow} r \frac{K+2}{2 K+1} \frac{\left\langle n u^{2}\right\rangle}{\langle n u\rangle}
$$

où $r=\bar{\tau}^{2}$; pour les collisions avec les phonons acoustiques, qui semblent prédominer dans le volume de l'échantillon à température ambiante $[11,12,13]$, $r=3 \pi / 8$. Les relations (11) et (12) permettent de déterminer $n_{1}$ et $\mu_{1}$ en fonction de $K$.

Le coefficient $K$ est fourni par les mesures de magnétorésistance longitudinale en fonction de l'induction ; pour simplifier les calculs théoriques (éq. (5)) nous avons utilisé le formalisme hydrodynamique (approximation $\tau(\varepsilon)=\mathrm{Cte}$ ); l'erreur qui en résulte ne dépasse pas $15 \%$ à température ambiante (et elle se trouve de plus compensée en partie par l'utilisation d'une large gamme d'inductions magnétiques).

Alors :

$$
\left(\frac{\rho_{\mathrm{B}}}{\rho_{0}}\right)_{110}^{110}=\frac{\langle n u\rangle}{\left\langle n u \frac{1+\frac{K+5}{2(2 K+1)} u^{2} B^{2}}{1+\frac{K+1}{2 K} u^{2} B^{2}}\right\rangle}
$$

La comparaison des courbes théoriques aux résultats expérimentaux est donnée en figure 6 , pour les deux modèles envisagés (et $r=3 \pi / 8$ ). Les résultats relatifs aux paramètres physiques obtenus sont résumés dans le tableau ci-contre pour deux des échantillons.

On constate que le coefficient d'anisotropie obtenu $(K \simeq 7)$ ne dépend pas du modèle utilisé ; si on considère que l'anisotropie de masse effective des couches de SSI est comparable à celle du silicium massif $\left(m_{1} / m_{\mathrm{t}} \simeq 4,7\right)$, on en déduit une anisotropie du temps de relaxation $\tau_{1} / \tau_{t} \simeq 0,67$.

L'écart le plus important entre les deux modèles concerne les mobilités de porteurs $\mu_{1}$ à l'interface $\mathrm{Si}_{-} \mathrm{SiO}_{2}$ : le modèle linéaire conduit à une valeur de $\mu_{1}$

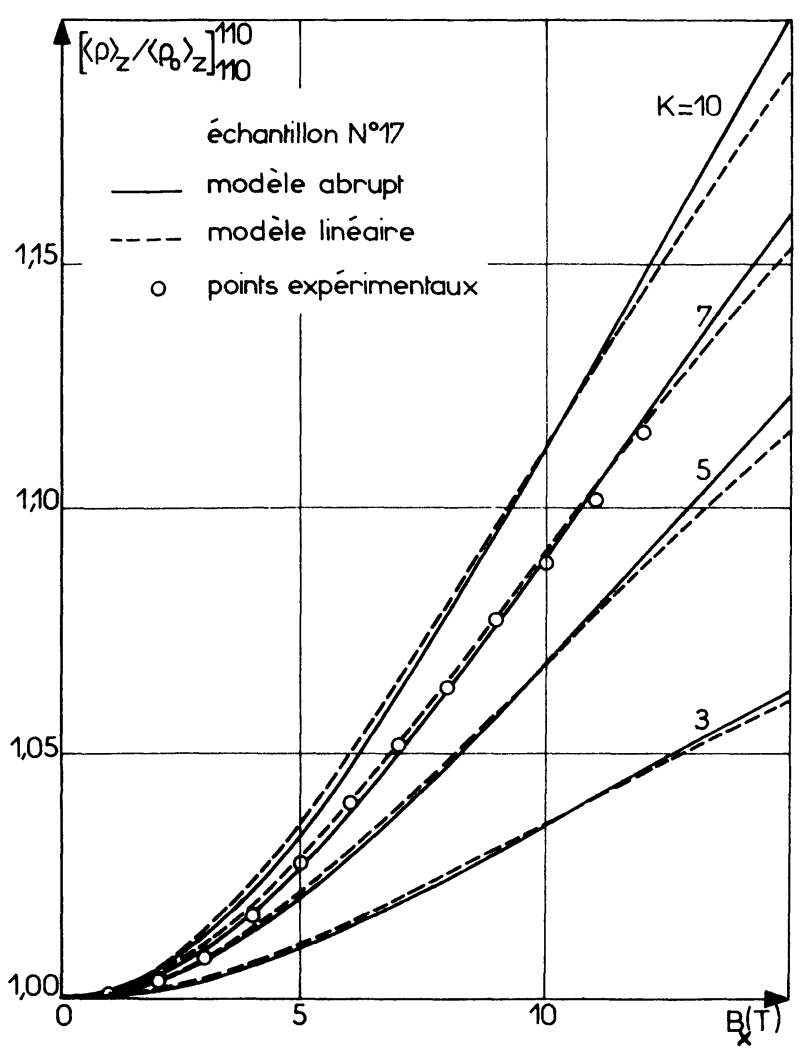

Fig. 6. - Coefficient de magnétorésistance longitudinale en fonction de l'induction magnétique $B_{X}$ pour diverses valeurs du coefficient d'anisotropie $K$. Les paramètres caractérisant les deux modèles sont définis dans le tableau I.

[Longitudinal magnetoresistance coefficient against the magnetic induction $B_{X}$ for several values of the anisotropy coefficient $K$. The main parameters of the two models are given in table $I$.

Tableau I. - Tahleau récapitulatif des résultats relatifs aux paramètres de transport des couches de Silicium Sur Isolant, obtenus pour chaque modèle (cf. Fig. 5) par effets galvanomagnétiques $;\langle\mu\rangle:$ mobilité moyenne des électrons dans la couche $; n_{1}, \mu_{1}$ : densité et mohilité des électrons à l'interface $\mathrm{Si}_{-} \mathrm{SiO}_{2}$. Les valeurs indiquées sont calculées en supposant prédominantes les collisions avec les phonons acoustiques $(r=3 \pi / 8)$; le cas hydrodynamique $r=1$ ne conduit qu'à des écarts inférieurs à $20 \%$.

[Transport parameters in Silicon On Sapphire films, as deduced from galvanomagnetic effects, for each model (cf. Fig. 5); $\langle\mu\rangle:$ mean electron mobility in the whole film; $n_{1}, \mu_{1}$ : electron density and mobility at $\mathrm{Si}-\mathrm{SiO}_{2}$ interface. These values have been calculated in the case of acoustical phonon scattering $(r=3 \pi / 8)$; for $r=1$, differences are lower than $20 \%$ ]

\begin{tabular}{cccc}
\multicolumn{5}{c}{ Modèle abrupt $: K \simeq 7$} \\
Echantillon & $\langle\mu\rangle\left(\mathrm{m}^{2} \mathrm{~V}^{-1} \mathrm{~s}^{-1}\right)$ & $\mu_{1}\left(\mathrm{Si}_{-} \mathrm{SiO}_{2}\right)$ & $n_{1}\left(\mathrm{~m}^{-3}\right)$ \\
- & - & - & - \\
$\mathrm{n}^{\circ} 1$ & 0,057 & 0,062 & $5,9 \times 10^{21}$ \\
$\mathrm{n}^{0} 17$ & 0,054 & 0,058 & $6,7 \times 10^{21}$ \\
\multicolumn{5}{c}{ Modèle linéaire $: K \simeq 7$} \\
$\mathrm{n}^{\mathbf{0}} 1$ & 0,043 & 0,093 & $7,9 \times 10^{21}$ \\
$\mathrm{n}^{0} 17$ & 0,040 & 0,088 & $8,9 \times 10^{21}$
\end{tabular}


relativement élevée $\left(\mu_{1} \simeq 0,09 \mathrm{~m}^{2} \mathrm{~V}^{-1} \mathrm{~s}^{-1}\right)$, mais qui demeure compatible avec les mesures rapportées pour d'autres couches de SSI [5]. Les mobilités moyennes dans la couche restent, elles, à peu près comparables. On ne peut donc révoquer aucun modèle puisque les diverses valeurs obtenues ne semblent pas irréalistes.

Pour pouvoir proposer un modèle pour les profils des paramètres de transport, ces mêmes effets galvanomagnétiques devront être mesurés sur des structures de diverses épaisseurs ou sur une même structure munie d'une grille de contrôle permettant de moduler l'épaisseur active du film. Elles seront éventuellement combinées à des mesures capacitives (ainsi qu'à des études en température permettant de préciser les processus de collision des porteurs).

5. Conclusion. - L'étude théorique que nous avons développée montre que les effets galvanomagnétiques classiques restent particulièrement utiles à l'analyse des semiconducteurs anisotropes présentant une inhomogénéité unidirectionnelle. Le cas des couches minces de Silicium Sur Isolant utilisées en microélectronique a été envisagé en priorité. Nous constatons qu'à partir des seules mesures de magnétorésistance longitudinale et d'effet Hall, nous pouvons déterminer de manière précise les paramètres d'anisotropie du matériau, ainsi que les propriétés de divers modèles plausibles d'inhomogénéité dans la direction de l'épitaxie et les valeurs des coefficients de transport importants (mobilité moyenne, densité et mobilité des porteurs à l'interface $\mathrm{Si}^{-} \mathrm{SiO}_{2}, \ldots$ )

Remerciements. - Nous tenons à remercier le LETI-MEA (CEN Grenoble), et plus spécialement Messieurs J. Borel, R. Staderini et J. de Pontcharra, pour la fourniture des échantillons de Silicium Sur Saphir et l'intérêt porté à cette étude dans le cadre du Groupement Circuits Intégrés sur Silicium (GCIS); nous exprimons notre gratitude au Professeur R. Pauthenet et à Monsieur Picoche qui nous ont permis d'utiliser les installations du Service National des Champs Intenses (SNCI, Grenoble) pour nos mesures à haut champ magnétique

\section{Bibliographie}

[1] Trilhe, J., Blanchard, B., Borel, J., Proc. 3rd Int. Conf. on Solid Surfaces, Vienna (Austria), 1977, 541.

[2] Cristoloveanu, S., Chovet, A., Kamarinos, G., Solid State Electron. 21 (1978) 1563; Rev. Phys. Appl. 13 (1978) 615.

[3] IPRI, A. C., J. Appl. Phys. 43 (1972) 2770.

[4] Hsu, S. T., ScotT, J. H., RCA Rev. 36 (1975) 240.

[5] Hsu, S. T., IEEE Trans. Electron. Devices ED 25 (1978) 913.

[6] BeER, A. C., Galvanomagnetic effects in Semiconductors (Academic Press, New York) Solid State Phys. Suppl. 4 (1963) p. 56
[7] Lippmann, H. J., KuhrT, F., Z. Naturforsch. 13a (1958) 462.

[8] Shibuya, M., Phys. Rev. 95 (1954) 1385.

[9] Vapaille, A., Physique des dispositifs à semiconducteurs. Electronique du Silicium homogène (Masson \& Cie Paris) (1970).

[10] Herring, C., Vogt, E., Phys. Rev. 101 (1956) 944.

[11] Elliot, A. B. M., Anderson, J. C., Solid State Electron. 15 (1972) 531

[12] Ross, E. C., Warfield, G., J. Appl. Phys. 40 (1969) 2339.

[13] Dumin, D. J., Ross, E. C., J. Appl. Phys. 41 (1970) 3139. 